\title{
One-step potentiostatic electrodeposition of polypyrrole/graphene oxide/ multi-walled carbon nanotubes ternary nanocomposite for supercapacitor
}

\begin{abstract}
ABSTRCT
A ternary nanocomposite consisting of polypyrrole (PPy), graphene oxide (GO) and multiwalled carbon nanotube (MWCNT) for supercapacitor was prepared via facile one step potentiostatic technique. Field emission scanning electron microscopy (FESEM) images displayed a randomly entangled nanostructure of MWCNT with rough wrinkle surface of GO incorporating with PPy granular structure represent the morphology of PPy/GO/MWCNT nanocomposite. The ternary nanocomposite was further justified its chemical composition by using Fourier transform infrared spectroscopy (FTIR) and Raman spectroscopy. $\mathrm{PPy} / \mathrm{GO} / \mathrm{MWCNT}$ exhibited a high specific capacitance of $358.69 \mathrm{~F} \mathrm{~g}-1$ at a scan rate of $100 \mathrm{mV} \mathrm{s}^{-1}$ in $1 \mathrm{M} \mathrm{Na} 2 \mathrm{SO}$, which is comparatively higher than both binary nanocomposites, PPy/MWCNT (207.52 F g-1) and PPy/GO (139.03 F g-1). The PPy/GO/MWCNT nanocomposite also possessed much longer charge-discharge time and excellent cycling stability $(88.69 \%)$ with the specific energy of $40.45 \mathrm{Wh} / \mathrm{kg}$ and specific power of $441.24 \mathrm{~W} / \mathrm{kg}$. Therefore, PPy/GO/MWCNT nanocomposite is a potential electrode material for high-performance supercapacitor.
\end{abstract}

Keyword: Ternary nanocomposite; Supercapacitor; Polypyrrole; Graphene oxide; Carbon nanotube. 